\title{
Reporte de hallazgo radiológico de hematoma intramural Duodenal posterior a uso de anticoagulantes
}

\author{
Report of radiological finding of duodenal intramural hematoma after use \\ of anticoagulants
}
Gema Gabriela Vera Loor. ${ }^{1}$, Steffania Renata Montesdeoca Santana. ${ }^{2}$, Marieta Plaza Salazar. ${ }^{3}$ \& Jéssica Bernabé Ruíz. ${ }^{4}$

\begin{abstract}
.
Introduction: The finding of intestinal intramural hematoma is rare, due to multiple factors, starting with its low incidence, 1 out of every 2500 patients receiving warfarin treatment. Its appearance is $90 \%$ associated with abdominal trauma, with few cases
\end{abstract}

\section{Resumen.}

Introducción: El hallazgo de hematoma intramural intestinal resulta rara, debido a múltiples factores, iniciando por su su baja incidencia, 1 de cada 2500 pacientes en tratamiento con warfarina. Su aparición se asocia en un 90\% a traumatismos abdominales, existiendo

1 Universidad de Guayaquil. Guayaquil, Ecuador. gemavera.30@gmail.com. (iD) ORCID https://orcid.org/0000-0002-1863-5309

2 Universidad Católica de Santiago de Guayaquil, Guayaquil, Ecuador. steff.montesdeoca@gmail.com. (iD) ORCID https://orcid.org/0000-0002-4817-227X

3 Universidad Católica de Santiago de Guayaquil. Guayaquil, Ecuador. marieta.plazas@gmail.com. ORCID https://orcid.org/0000-0007-9264-3123

4 Universidad de Guayaquil. Guayaquil, Ecuador. jekbernar@hotmail.com. (iD) ORCID https://orcid.org/0003-3367-0361 
reported in relation to anticoagulant overdose. It is a highly infrequent cause of acute abdominal pain, but it becomes a differential diagnosis to be taken into account in a patient with a history of anticoagulation. In spontaneous cases, its location is more frequent in the jejunum (71.6\%) followed by the duodenum (29.8\%). Objective: To report this rare radiological finding. Methodology: In this article we detail the rare radiological finding by computed tomography of an intramural hematoma in the small intestine specifically in the second and third portion of the duodenum related to anticoagulation; from the report of a reported clinical case. Conclusion: The report of radiological findings of rare entities such as non-traumatic duodenal intramural hematoma is important and nutritious in terms of scientific evidence, expanding the range of options in patients with unspecified abdominal pain with suspected anticoagulation overdose.

Keywords: hematoma, intramural, intestinal, computed tomography. pocos casos reportados en relación a sobredosis de anticoagulantes. Es causa altamente infrecuente de dolor abdominal agudo, pero se convierte en un diagnóstico diferencial a tomar en cuenta en paciente con antecedentes de anticoagulación. En casos espontáneos su localización es más frecuente en el yeyuno $(71.6 \%)$ seguido del duodeno (29.8\%). Objetivo: Reportar este raro hallazgo radiológico. Metodología: En el presente artículo detallamos el raro hallazgo radiológico mediante tomografía computarizada de un hematoma intramural en intestino delgado específicamente en segunda y tercera porción del duodeno relacionado a anticoagulación; a partir del relato de un caso clínico reportado. Conclusión: El reporte de hallazgos radiológicos de entidades raras como lo es el hematoma intramural duodenal no traumático es importante y nutritivo en cuanto a evidencia científica, ampliando el abanico de opciones en pacientes con dolor abdominal no especificado con sospecha de sobredosis de anticoagulación

Palabras claves: hematoma, intramural, intestinal, tomografía computarizada

\section{Introducción}

La presentación del hematoma intramural intestinal es una entidad poco habitual, debida a su baja incidencia (Flores \& Morales-Barroso, 2015), resultando en 1 de cada 2500 pacientes en tratamiento con warfarina (Miras et al., 2016; Arnáiz et al., 2006). Consiste en la acumulación de sangre en la pared intestinal y generalmente su aparición se asocia en un $90 \%$ a traumatismos abdominales; al igual que los hematomas intramurales de colon (Wang \& Sun, 2020; Sheng-Wen et al., 2008), es poco habitual una complicacion por la utilizacion de anticoagulantes orales (Folgado \& Sánchez, 2008), o a enfermedad hematológica de base con cifras de incidencia no disponibles (Reyes \& Tagle, 2010). Se presenta con mayor frecuencia en hombres que en mujeres, y su edad media de 58 años, varios grados de dolor abdominal agudo con signos peritoneales harían un diagnóstico 
engorroso (Bekheit \& AllaaSallan, 2014); pero se convierte en un diagnóstico diferencial a tomar en cuenta en paciente con antecedentes de anticoagulación (García-Marín \& García-Marín, 2009). En casos espontáneos su localización es más frecuente en el yeyuno (71.6\%) seguido del duodeno (29.8\%), siendo contrario su localización en postraumáticos. La clínica resulta confusa y genérica ya que sus síntomas resultan muy inespecíficos (p. ej. dolor abdominal y náuseas), provocando con facilidad errores diagnósticos (Sheng-Wen et al., 2008); por lo que debe existir una alta sospecha clinica teniendo en cuenta la tecnica de elección a la TC para el disgnostico y seguimiento (García-Espinoza et al., 2017). Convirtiéndose los hallazgos radiológicos en nuestros mejores aliados, con mayor especificidad la tomografía computarizada (Miras et al., 2016), por este motivo nos enfocamos en presentar el hallazgo radiológico de esta rara entidad a partir del caso clínico a continuación relatado.

\section{Metodología}

Presentamos hallazgo radiológico a propósito del caso clínico de paciente masculino de 67 años quien refiere como antecedente hipertensión arterial y accidente cerebro vascular medicado con anticoagulantes orales hace 1 año, sin secuelas motoras aparentes al momento, que acude al servicio de emergencia por presentar cuadro clínico de aproximadamente 5 horas de evolución caracterizada por dolor precordial, frialdad, sudoración profusa, acompañado de taquicardia e hipotensión, por lo que se maneja como síndrome coronario agudo con carga de anticoagulantes orales: aspirina y clopidogrel, e ingreso hospitalario a unidad de cuidados intensivos para tratamiento especializado y monitoreo continuo. Luego de 48 horas posteriores a su ingreso presenta melena por lo que se sospecha de hemorragia digestiva alta, se da orden de nada por vía oral (NPO); se realiza endoscopía alta que reporta: bulbitis erosiva grado moderado, úlceras bulbares activas; una con vaso visible, úlceras superficiales activas prepilóricas, gastropatía erosiva grado moderada con cambios atróficos moderados, lesiones de tipo polipoidea de cuerpo y techo gástrico, se realiza esclerosis (fig.1). Dos días después de mencionado procedimiento exámenes hemáticos complementarios marcan descenso marcado de hematocrito de $36.1 \%$ a $25.8 \%$ y hemoglobina de $12.0 \mathrm{~g} / \mathrm{dl}$ a $8.9 \mathrm{~g} / \mathrm{dl}$, por lo que se procede a la administración de derivados hemáticos, ácido tranexánico y vitamina $\mathrm{k}$, además se realiza ecografía abdominal de control donde se observa formación hipoecogénica alargada que mide $10 \times 5 \mathrm{~cm}$ aproximadamente, en topografía del duodeno; que se reporta como masa abdominal duodenal vs hematoma intramural duodenal a investigar. Debido a hallazgo ecográfico y presencia de dolor abdominal persistente en paciente se procede a realizar tomografía computarizada de abdomen donde hallamos engrosamiento hiperdenso concéntrico y homogéneo de pared intestinal de segunda-tercera porción de duodeno dando el diagnóstico definitivo de hematoma intramural intestinal (fig. 2).

\section{Discusión}

El hematoma intramural intestinal es una entidad de rara presentación, consiste en la acumulación de sangre en la pared intestinal (Miras et al., 2016; Martínez et al., 2005); 
presenta baja incidencia, resultando en 1 de cada 2500 pacientes en tratamiento con warfarina (Miras et al., 2016; Arnáiz et al., 2006). Generalmente se asocia en un $90 \%$ a traumatismos abdominales, aunque en pocos casos reportados se encuentra relacionado a sobredosis de anticoagulantes o a enfermedad hematológica de base con cifras de incidencia desconocidas (Reyes \& Tagle, 2010). Es más frecuente en hombres y la edad media de presentación es 58 años, es causa altamente infrecuente de dolor abdominal agudo, pero se convierte en un diagnóstico diferencial a tomar en cuenta en pacientes con antecedentes de anticoagulación (Carvajal et al., 2006). En casos espontáneos su localización más frecuente es en el yeyuno (71.6\%) seguido del duodeno (29.8\%), siendo contraria la localización en postraumáticos. Sus síntomas son muy inespecíficos por ejemplo dolor abdominal en el 90,7\%, náuseas en un 50\%, provocando con facilidad errores diagnósticos. Convirtiéndose los hallazgos radiológicos en nuestros mejores aliados, con mayor especificidad la Tomografía computarizada (Carvajal et al., 2006; Sheng-Wen et al., 2008). La ecografía en general tiende a ser la primera herramienta empleada para el estudio del dolor abdominal, pero resulta un método muy sensible pero poco específico, ya que los hallazgos como engrosamiento hiperecogénico de la capa submucosa en dicho segmento intestinal o disminución del peristaltismo pueden estar presentes en otras entidades como la colitis infecciones o isquémicas; y enfermedad inflamatoria intestinal, no quitando el valor añadido como prueba de primer nivel para investigación de causa desconocida de dolor abdominal, que acompañado de una adecuada historia clínica debería motivarnos a la realización de más estudios complementarios para su estudio (Azcón et al., 2014; Sheng-Wen et al., 2008). Por otro lado, la tomografía computarizada toma importante atención ya que es la modalidad de elección para el diagnóstico de esta rara entidad. Entre sus principales y relevantes hallazgos encontramos: engrosamiento circunferencial, simétrico e hiperdenso de la pared de un segmento intestinal, puede estar presente también la hemorragia intraluminal, peritoneal o en el meso del asa afectada (Sheng-Wen et al., 2008; Polat et al., 2003). En cuanto al tratamiento muchos autores aseguran el consenso sobre un abordaje inicialmente conservador que conlleva generalmente a la resolución del cuadro clínico en un promedio entre 10-15 días aproximadamente, requiriendo la administración de vitamina $\mathrm{k}$, derivados hemáticos e interrupción de anticoagulación en casos secundarios a la misma. Reservando el tratamiento invasivo o quirúrgico en casos de isquemia intestinal, perforación, hemorragia intraluminal activa, fallo del tratamiento conservador, dudas diagnósticas, complicaciones tardías o recurrencias; éstas dos últimas muy infrecuentes (Cadavid et al., 2009).

\section{Conclusión}

Concluimos que el reporte de hallazgos radiológicos de entidades raras como lo es el hematoma intramural duodenal no traumático es importante y nutritivo en cuanto a evidencia científica, ampliando el abanico de opciones en pacientes con dolor abdominal no especificado con sospecha de sobredosis de anticoagulación. Porque, aunque clínicamente esta entidad es inespecífica, los hallazgos radiológicos y antecedentes se convertirán en nuestros mayores aliados para el seguimiento y diagnóstico definitivo. En 


\section{ADDigital}

ISSN: 2697-3391

casos de evolución tórpida, complicación y recurrencia el tratamiento quirúrgico se convierte en un tema obligatorio, no siempre asegurando los mejores resultados.

\section{Figura 1}

\section{Imágenes de endoscopía alta}

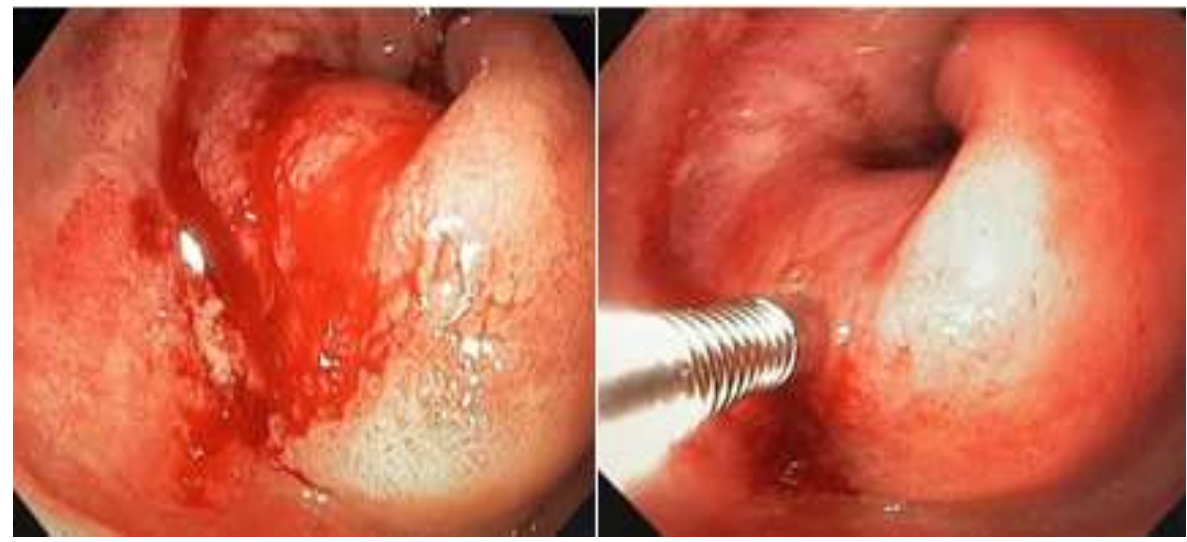

Nota: Imágenes de endoscopía alta: (Izquierda) Bulbitis erosiva grado moderado úlceras bulbares activas; una con vaso visible, úlceras superficiales activas prepilórica, gastropatía erosiva grado moderada con cambios atróficos moderados, lesiones de tipo polipoidea de cuerpo y techo gástrico. (Derecha) Posterior a realizar esclerosis.

\section{Figura 2}

Tomografía computarizada de abdomen corte axial y transversal

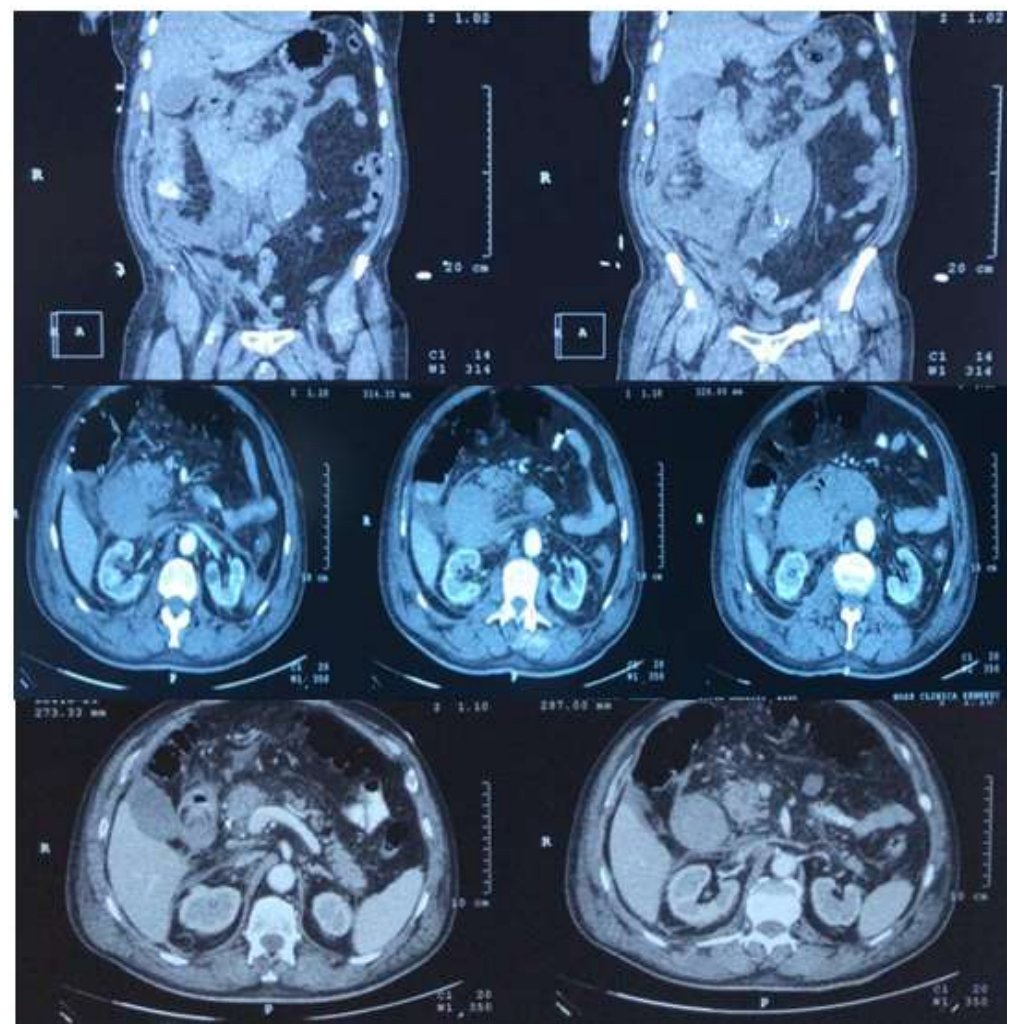

Nota: Tomografía computarizada de abdomen corte axial y transversal:

engrosamiento hiperdenso concéntrico y homogéneo de pared intestinal de segunda-tercera porción de duodeno compatible con hematoma intramural intestinal. 


\section{Referencias bibliográficas}

Arnáiz, A., Arnáiz, J. \& Marco de Lucas, E., (2006), Dolor abdominal en paciente tratado con acenocumarol. Un caso de hematoma intramural espontáneo de intestino delgado; Anales de Medicina Interna.

Azcón, F., Martínez, A. \& Santiago, A. (2014). Hematoma intramural intestinal espontáneo: espectro de presentación y diagnóstico por imagen. SERAM.

Cadavid, A., Uribe, J. \& Mesa, M. (2009). Hematoma espontáneo del intestino delgado. Reporte de dos casos y revisión de la literatura; Revista Colombiana de Cardiología.

Carvajal, J., Casado, J. \& Martín, M. (2006). Hematoma espontáneo de la pared abdominal asociada a la terapia antiagregante en el paciente geriátrico; Sociedad Española de Geriatría y Gastroenterología.

Folgado, A. S. \& Sánchez, P. (2008). Hematoma intramural espontáneo no traumático del intestino delgado: una complicación poco habitual de los anticoagulantes

Flores, A. \& Morales-Barroso, M., (2015). Hematoma intramural gástrico en paciente no anticoagulado. Revista Andaluza de Patología Digestiva (RAPD).

García-Marín, A., A. \& García-Marín, J. (2009). Hematoma intramural espontáneo de ciego. Revista Española de enfermedades digestivas. Elsevier.

García-Espinoza, J., Martínez-Martínez, A. \& Medina-Benítez, A. (2017). Hematoma intramural duodenal secundario a tratamiento endoscópico en paciente con enfermedad hematológica. Revista Andaluza de Patología Digestiva (RAPD).

Martínez, J., Rey, M., Marulanda, J. \& Garzón, M. (2005). Hematoma intramural esofágico. Revista Colombiana de Gastroenterología.

Miras, A., Martínez, A. \& Santiago, A. (2016). Hallazgo en imágenes de hematoma intramural espontáneo; Revista Argentina de Radiología.

Bekheit, M. \& AllaaSallan, M. (2014) Non-traumatic intramural hematomas in patients on anticoagulant therapy: Report of three cases and overview of the literature. African Journal of Emergency Medicine.

Polat, C., Dervisogl, A., Guven, H. \& Kaya, E. (2003). Anticoagulant-induced intramural intestinal hematoma. The American Journal of emergency medicine.

Reyes, H. \& Tagle, M. (2010). Hematoma intramural espontáneo de intestino delgado debido al uso de anticoagulantes orales: Reporte de caso y revisión de literatura; Revista de Gastroenterología de Perú.

Sheng-Wen, Hou., Chien-Chih, Chen. \& Kuo-Chi,h Chen. (2008). Sonographic diagnosis of spontaneous intramural small bowel hematoma in a case of warfarin overdose; Journal of clinical ultrasound. 
Wang, Jing. \& Sun, Xiaoyan. (2020) Hematoma intramural of colon. Medicine.

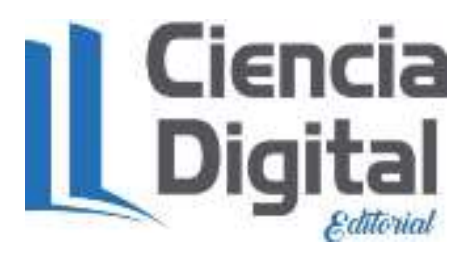




\section{PARA CITAR EL ARTÍCULO INDEXADO.}

Vera Loor, G. G., Montesdeoca Santana, S. R., Plaza Salazar, M., \& Bernabé Ruíz, J. (2021). Reporte de hallazgo radiológico de hematoma intramural Duodenal posterior a uso de anticoagulantes. Anatomía Digital, 4(4), 159-166. https://doi.org/10.33262/anatomiadigital.v4i4.1949

\section{LCiencia}

El artículo que se publica es de exclusiva responsabilidad de los autores y no necesariamente reflejan el pensamiento de la Revista Anatomía Digital.

El artículo queda en propiedad de la revista y, por tanto, su publicación parcial y/o total en otro medio tiene que ser autorizado por el director de la Revista Anatomía Digital.
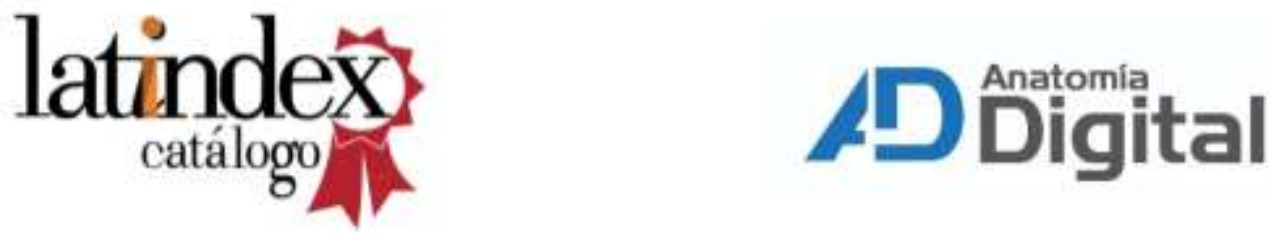